\title{
Mission Assessment of the Faraday Accelerator with Radio-frequency Assisted Discharge (FARAD)
}

\author{
John W. Dankanich* \\ Gray Research, Inc., Huntsville, AL, 35806 \\ and \\ Kurt A. Polzin ${ }^{\dagger}$ \\ NASA Marshall Space Flight Center, Huntsville, AL, 35812
}

\begin{abstract}
Pulsed inductive thrusters have typically been considered for future, high-power, missions requiring nuclear electric propulsion. These high-power systems, while promising equivalent or improved performance over state-of-the-art propulsion systems, presently have no planned missions for which they are well suited. The ability to efficiently operate an inductive thruster at lower energy and power levels may provide inductive thrusters near term applicability and mission pull. The Faraday Accelerator with Radio-frequency Assisted Discharge concept demonstrated potential for a high-efficiency, low-energy pulsed inductive thruster. The added benefits of energy recapture and/or pulse compression are shown to enhance the performance of the pulsed inductive propulsion system, yielding a system that con compete with and potentially outperform current state-of-the-art electric propulsion technologies. These enhancements lead to mission-level benefits associated with the use of a pulsed inductive thruster. Analyses of low-power near to mid-term missions and higher power farterm missions are undertaken to compare the performance of pulsed inductive thrusters with that delivered by state-of-the-art and development-level electric propulsion systems.
\end{abstract}

\section{Introduction}

For decades, high-power pulsed plasma thrusters have claimed equivalent or improved performance over state-of-the-art (SOA) steady-state electric propulsion (EP) systems. After considerable work the Northrop-Grumman Space Technology (formerly TRW) Pulsed Inductive Thruster (PIT), which represents the SOA in inductive thruster technology, has obtained relatively high performance in the laboratory environment. ${ }^{1}$ However, it still requires additional advancements in switching technology and energy storage before becoming practical for high-power in-space applications. While high power pulsed systems have performance advantages over SOA systems, these systems-level limitations mean that there are currently few planned missions where this high-power thruster could even be applicable. A high-performance pulsed inductive thruster operating at lower power could be used on many planned missions, overcoming several of the technical issues associated with higher-power operation and permitting greater near-term acceptance of the technology. The Faraday Accelerator with Radio-frequency Assisted Discharge (FARAD) ${ }^{2}$ is a lower-power alternative to the PIT that has the potential for in-space operation using current SOA power storage and switching. In the present paper we investigate a range of science and exploration missions (at both low and high power) to find the niche that is best suited for pulsed inductive thrusters. While this study is specifically focused on FARAD, the methodology and general conclusions should extend to other pulsed inductive accelerators like the PIT and compact toroid-based thrusters. ${ }^{3}$

\footnotetext{
* Systems Engineer, NASA’s In-Space Propulsion Technology Project, Member AIAA.

$\dagger$ Propulsion Research Engineer, Propulsion Research and Technology Applications Branch, Propulsion Systems Department, Senior Member AIAA.
} 


\section{FARAD}

\section{A. Thruster Concept}

In the FARAD thruster, shown in Fig. 1, energy is stored in a capacitor and then discharged through an inductive acceleration coil. The device is electrodeless, inducing current in a plasma located near the face of the coil. The propellant is accelerated and expelled at a high exhaust velocity $(\sim 10 \mathrm{~km} / \mathrm{s})$ through the interaction of the plasma current and the induced magnetic field. FARAD is similar to the PIT in that both use current flowing through an inductive coil to accelerate the plasma. In the PIT, both propellant ionization and acceleration are performed by the pulse of current in the coil. In contrast, FARAD uses a separate inductive RF discharge to preionize the propellant before it is accelerated by the current pulse. This preionization allows FARAD to operate at much lower discharge energies than the PIT (order of 100 $\mathrm{J} /$ pulse vs. $4 \mathrm{~kJ} /$ pulse), and allows for a concomitant reduction in the thruster's switching

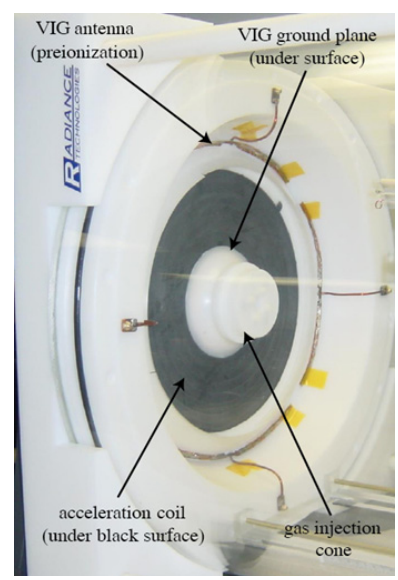

(a)

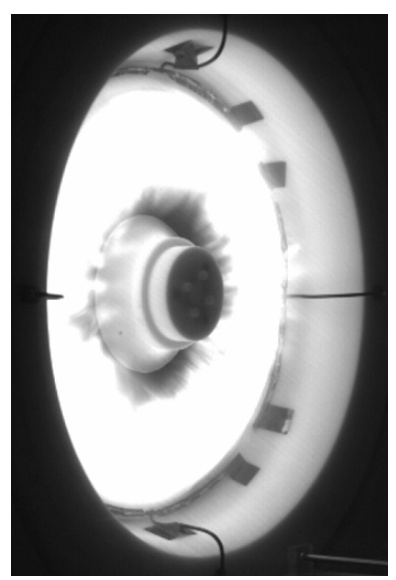

(b)

Figure 1. a) Photograph of the FARAD laboratory-model thruster coupled to a vacuum chamber and b) time-integrated argon ion light emission (488 $\mathrm{nm}$ line) from a single shot of the FARAD operating at a discharge energy of $100 \mathrm{~J}$. and energy storage requirements. A recent review of pulsed inductive thruster research and technology, including efforts to address these requirements, can be found in the archival literature. ${ }^{4}$

\section{B. Performance}

In the present study, a FARAD thruster operating at a discharge energy of $100 \mathrm{~J} /$ pulse, a frequency of $400 \mathrm{~Hz}$, and a mass bit of $0.15 \mathrm{mg} / \mathrm{pulse}$ is assumed to possess a thrust efficiency of $50 \%$ and a specific impulse, $I_{\mathrm{sp}}$, of $2500 \mathrm{~s}$. This level of performance is comparable with the SOA PIT and represents a developmental goal for FARAD. ${ }^{5}$ The propulsion system's front-end powertrain efficiency, consisting of the power electronics providing energy from the power source to the capacitors, is assumed to be $90 \%$. In addition, the Bernardes and Merryman $(\mathrm{B} \& \mathrm{M})^{6}$ and pulse compression $\mathrm{B} \& \mathrm{M}(\mathrm{PCBM})^{7}$ power trains have the capability to recapture energy not used in a pulse and apply it to subsequent pulses. ${ }^{8}$ A summary of the assumed performance of a FARAD thruster system is presented in Table $\mathrm{I}$.

Table I. Summary of FARAD performance assumptions.

\begin{tabular}{|l|c|l|}
\hline Energy Per Pulse & 100 & Joules \\
\hline Mass bit & 0.15 & $\mathbf{m g}$ \\
\hline Specific Impulse & 2500 & s \\
\hline Thrust efficiency & $50 \%$ & \\
\hline PowerTrain Efficiency & $90 \%$ & \\
\hline Bus Voltage to Powertrain Input Efficiency & $100 \%$ & \\
\hline Recapture Efficiency & $60 \%$ & \\
\hline Repetition Limit & 400 & $\mathrm{~Hz}$ \\
\hline
\end{tabular}




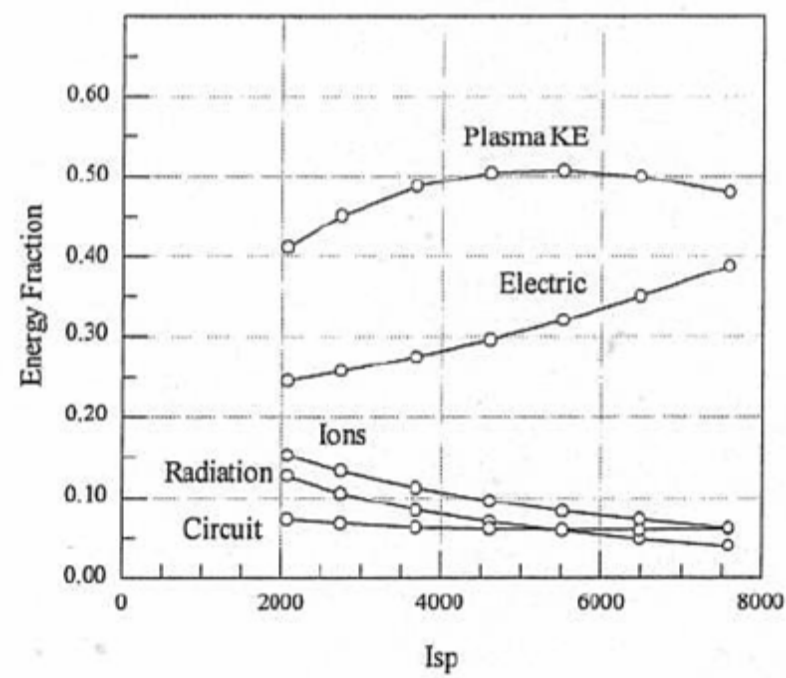

Figure 2. Energy Partitioning in PIT.

An energy recapture efficiency of $60 \%$ is assumed for the purpose of this study, but this may underestimate the level that can be recaptured and applied to subsequent pulses. Figure 2 is an illustration of energy partitioning in the PIT. The "electric" curve represents the energy remaining in the capacitor bank at the end of a pulse. The "ion" energy is lost due to ion heating during the snowplow phase of the plasma acceleration. "Radiation" energy losses are due to light emission from the plasma, while resistive heating is the source of the "circuit" energy sink. Based on the TRW PIT study ${ }^{9}$, it is estimated that of the energy not converted to plasma kinetic energy, $43 \%$ is available for recovery; $26 \%$ of the total energy input to the thruster. As the figure shows, a higher percentage of energy may be available for recapture at higher $I_{s p}$ levels

(approaching 75\%).

The overall efficiency of the FARAD system can be calculated, as shown in Table II, by taking the ratio of directed kinetic energy to the energy that must be expended by the power source to charge the system prior to a pulse. The powertrain efficiency of $90 \%$ implies that to charge the capacitor bank to $100 \mathrm{~J}$, a total of $111.11 \mathrm{~J}$ must be delivered by the charging power source. If the thruster was operating without any energy recapture (single pulse mode), $111.11 \mathrm{~J}$ would be required for every pulse generating 50 Joules of kinetic energy. Since the thruster is $50 \%$ efficient, the total system efficiency in this case is $45 \%$.

Table II. System efficiency per pulse of the baseline FARAD thruster.

\begin{tabular}{|l|c|c|c|c|c|c|c|c|}
\cline { 2 - 8 } \multicolumn{1}{c|}{} & Source Energy, J & Powertrain Eff. & Energy into Thruster, J & Thrust Eff. & Thruster KE, J & Recovered Energy, J & Energy Spent, J & System Eff. \\
\hline Single & 111.11 & $90 \%$ & 100 & $50 \%$ & 50 & 0 & 111.11 & $45.00 \%$ \\
\hline Initial & 111.11 & $90 \%$ & 100 & $50 \%$ & 50 & 30 & 81.11 \\
\hline Subsequent & 81.11 & $90 \%$ & 100 & $50 \%$ & 50 & 30 & $81.00 \%$ \\
\hline
\end{tabular}

Using energy recapture, only the first (initial) pulse requires $111.11 \mathrm{~J}$ from the power supply. At a recapture efficiency of $60 \%, 30 \mathrm{~J}$ are recovered and available for use in subsequent pulses, lowering the energy per pulse demanded from the charging supply to $81.11 \mathrm{~J}$. Continuous operation in the energy recovery mode increases the overall efficiency asymptotically to the maximum efficiency of $\sim 62 \%$. The overall system efficiency as a function of the number of pulses is presented in Fig. 3. Since even a relatively low $\Delta \mathrm{V}$ mission requires an estimated $10^{7}$ pulses, we are safe in using the asymptotic system efficiency value in the present study.

Pulsed thrusters, unlike steady-state plasma thrusters, do not require a continuous minimum power level to maintain a stable discharge. The capacitor bank can be charged over an arbitrarily long or short time interval. The only requirement is

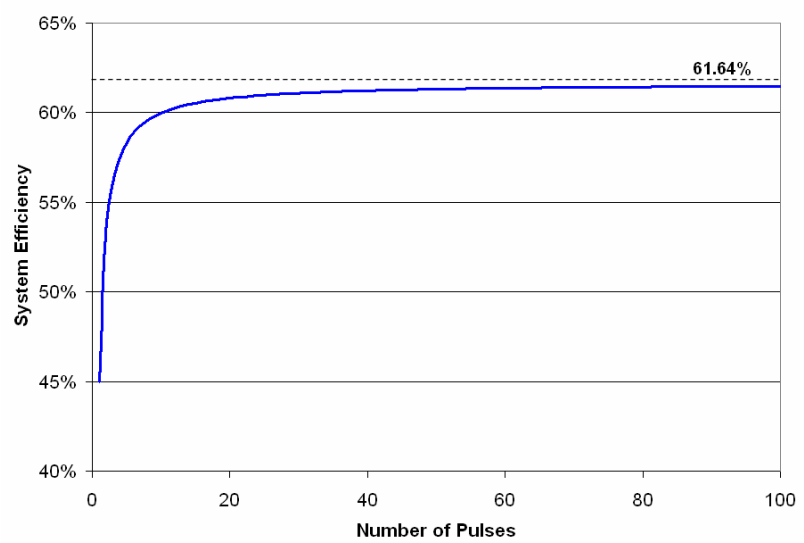

Figure 3. System Efficiency as function of number pulses. 

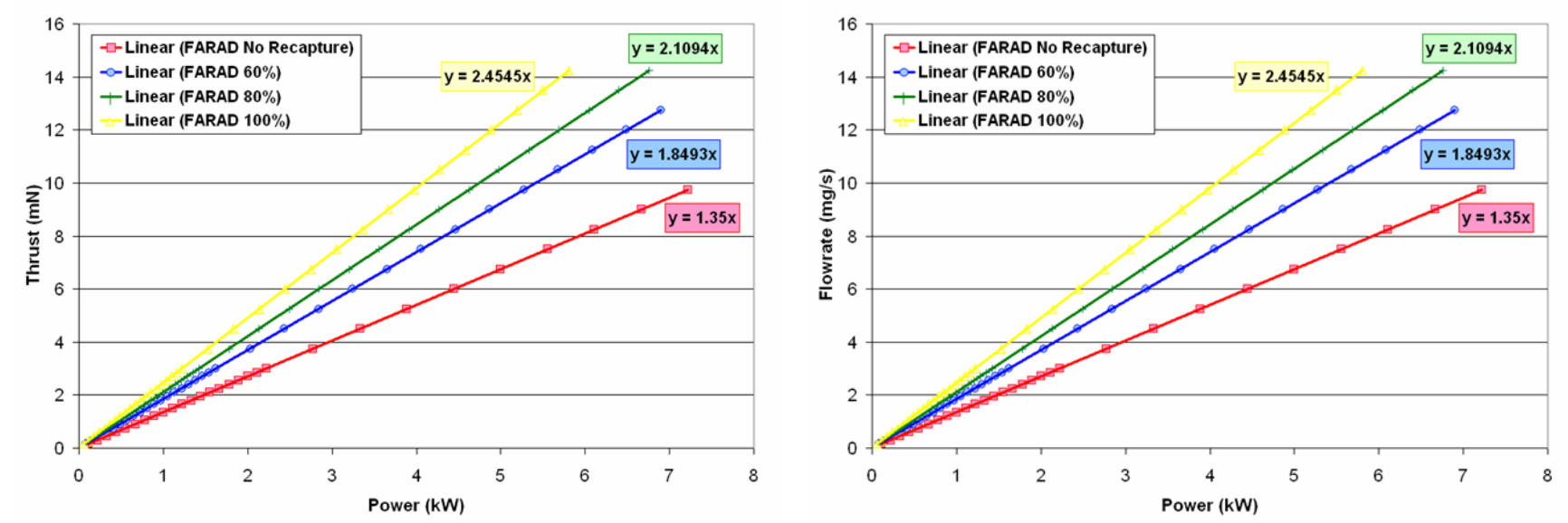

Figure 4. Thrust (left) and flowrate (right) of the FARAD as a function of power.

that the interval must be short enough to permit a pulse rate commensurate with the thrust level required for a given mission. The FARAD operating at a maximum pulse rate of $400 \mathrm{~Hz}$ and a discharge energy of $100 \mathrm{~J} /$ pulse would consume $40 \mathrm{~kW}$ (not including the powertrain losses), which we assume is possible to generate given SOA or near-term switching and power processing technology. A typical low-thrust analysis requires polynomial functions representing the thrust and propellant flowrate of the system as a function of power. Linear functions representing the thrust and flowrate in a FARAD thruster are presented in Fig. 4, with the energy recapture efficiency varied parametrically to generate a family of curves in each graph.

\section{System Mass}

Another attribute critical to the performance of a propulsion system is the dry system mass. The components of an overall system include the thruster, power processing unit, and propellant feed system. Mass breakdown estimates are provided in Table III for thruster systems employing either a B\&M or PCBM powertrains. ${ }^{10}$

In addition to the thruster system masses, a charging power supply must also be included in the overall mass budget. For this study, it is assumed that the charging power supply has a specific power of 6 $\mathrm{kg} / \mathrm{kW}$. The total FARAD system specific power will vary with maximum operating power. the charging power supply is sized for the maximum input power, capacitors and other hardware scale with both maximum power (for heat loads) and energy per pulse, which will raise the

Table III. System mass for B \& M (left) and PCBM (right) systems.*

\begin{tabular}{|c|c|}
\hline Capacitors & 9.2 \\
\hline Thruster Coil & 0.4 \\
\hline Coil Connectors & 0.2 \\
\hline Coil Base (Macor) & 5.0 \\
\hline Magnetic Switches & NA \\
\hline Preionizer, Antenna, Cabling & 10.0 \\
\hline Valve & 0.6 \\
\hline Feed Associated Apparatus & 1.0 \\
\hline Solid State Switches & 5.1 \\
\hline Bus Work (Copper) & 1.4 \\
\hline Diodes & 2.1 \\
\hline Coaxial Cables & 1.3 \\
\hline Control Electronics & 5.0 \\
\hline Structural \& Housing & 10.0 \\
\hline Total & $51.3 \mathrm{~kg}$ \\
\hline
\end{tabular}

\begin{tabular}{|c|c|}
\hline Capacitors & 13.8 \\
\hline Thruster Coil & 0.4 \\
\hline Coil Connectors & 0.2 \\
\hline Coil Base (Macor) & 5.0 \\
\hline Magnetic Switches: $1-1 "$ + 2-2" & 42.5 \\
\hline Preionizer, Antenna, Cabling & 10.0 \\
\hline Valve & 0.6 \\
\hline Feed Associated Apparatus & 1.0 \\
\hline Solid State Switches & 8.0 \\
\hline Bus Work (Copper) & 2.8 \\
\hline Diodes & 1.1 \\
\hline \begin{tabular}{|l|} 
Coaxial Cables \\
\end{tabular} & 1.3 \\
\hline Control Electronics & 5.0 \\
\hline Structural \& Housing & 10.0 \\
\hline Total & $101.7 \mathrm{~kg}$ \\
\hline
\end{tabular}

*Exclusing Charging Power Supply system alpha when operating at lower than maximum repetition rate. For a $10 \mathrm{~kW}$ system, the total specific powers are $11.13 \mathrm{~kg} / \mathrm{kW}$ and $16.17 \mathrm{~kg} / \mathrm{kW}$ for the B\&M and PCBM powertrains, respectively. The improvement in system specific mass (system alpha) as a function of input power is illustrated in Fig. 5. Due to discharge energy and pulse rate limitations, additional thrusters and their associated subsystems are required to process input 
power levels greater than the $40 \mathrm{~kW}$. This effect of adding more FARAD thruster systems to the spacecraft can be seen in Fig. 5 as a series of discontinuities in specific power.

\section{SOA Electric Propulsion Systems}

Research and development of electric propulsion thruster systems for NASA science missions have primarily been focused on griddedion engines and Hall thrusters. Ion engines provide higher throughput capability, higher specific impulse, and higher thrust efficiency relative to other EP systems. Hall thrusters are a proven, robust alternative, providing the benefits of simplicity

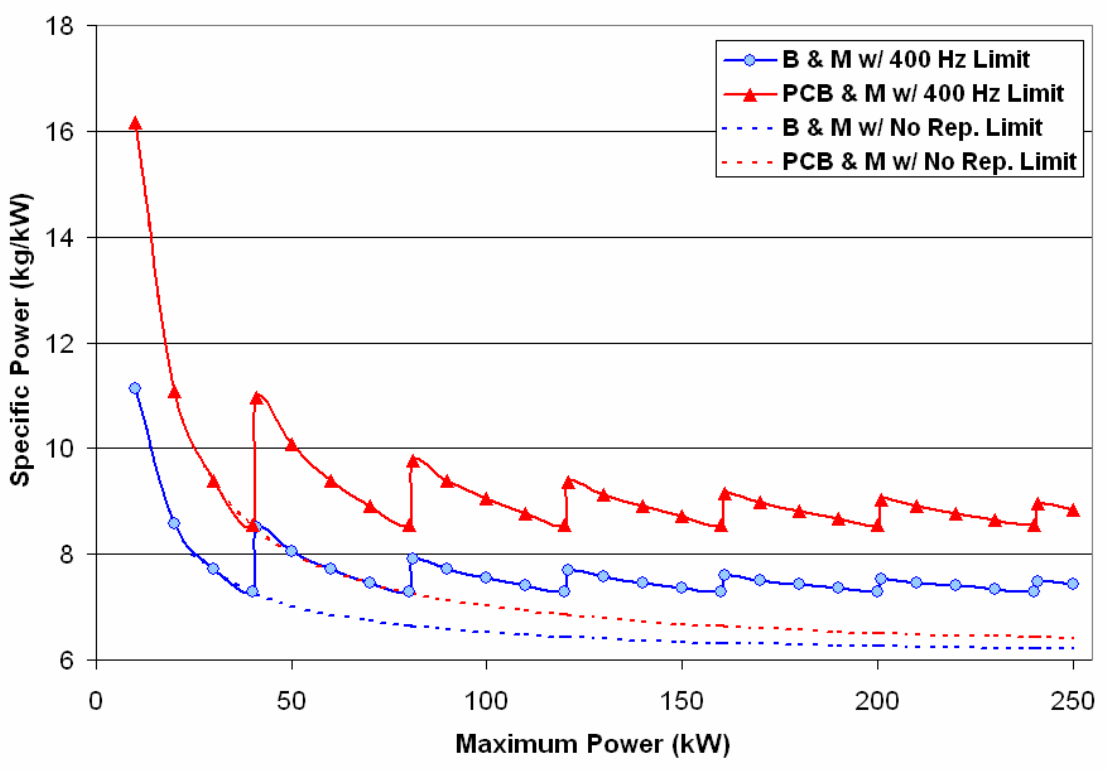

Figure 5. FARAD system alpha as a function of maximum operating power. and relatively low cost, and have thrust densities that exceed those produced by ion engines. In comparison with gridded-ion engines and Hall thrusters, FARAD has an advantage in that it is electrodeless, eliminating the lifetime and contamination issues associated with electrode erosion. Also, FARAD is throttleable in the sense that for a fixed energy per pulse it can maintain the same performance over a wide range of input power levels through adjustment of the pulse rate. In contrast steady-state thrusters exhibit decreasing performance as the input power is reduced. They also require a minimum input power level to sustain a stable electric discharge.

The specific SOA thrusters under consideration for this comparative study are the NASA Solar electric propulsion Technology Application Readiness (NSTAR) ion thruster, NASA's Evolutionary Xenon Thruster (NEXT), the NASA-103M High Voltage Hall Accelerator (HiVHAC) and the commercial BPT-4000 Hall thruster. These thrusters were chosen because they have the potential to meet NASA's current science mission propulsion needs. ${ }^{11}$ Each thruster's characteristics and nominal performance values are presented in Table IV. While the thrusters can operate over a large range of conditions, as indicated by the throttle curves presented in Fig. 6, the performance values listed in the

Table IV. Thruster Characteristics.

\begin{tabular}{|l|c|c|c|c|}
\cline { 2 - 5 } \multicolumn{1}{c|}{} & NSTAR & BPT 4000 & NEXT & HiVHAC \\
\hline Max. Input Power (kW) & 2.3 & 4.5 & 6.9 & 3.6 \\
\hline Throttle Range & $4.8: 1$ & $4.5: 1$ & $12.6: 1$ & $12: 1$ \\
\hline Specific Impulse (s) & 3,100 & 2,152 & 4,170 & 2,750 \\
\hline Max. Thrust (mN) & 94 & 278 & 236 & 151 \\
\hline Efficiency at Full Power & 0.6 & 0.6 & 0.7 & 0.57 \\
\hline Thrust to Power (mN/kW) & 40 & 62 & 34 & 42 \\
\hline Throughput (kg) & 157 & $>181$ & $>300$ & $>300$ \\
\hline Specific Mass (kg/kW) & 3.6 & 2.73 & 1.9 & 2.4 \\
\hline
\end{tabular}

*Predictions for BPT-4000, NEXT, and HiVHAC far exceed these numbers. NSTAR and BPT-4000 are fully qualified, NEXT is PM unit and HiVHAC is development unit. table are used in the present study. Note that systems efficiencies shown are lower than the thruster efficiencies due to PPU losses. The propellant throughput (lifetime) quoted for the NSTAR thruster is based on a completed life test, while the NEXT and HiVHAC values represent the objectives of the developmental effort. The BPT-4000's current qualified throughput is listed in the table, but additional testing is underway to improve upon that value. 

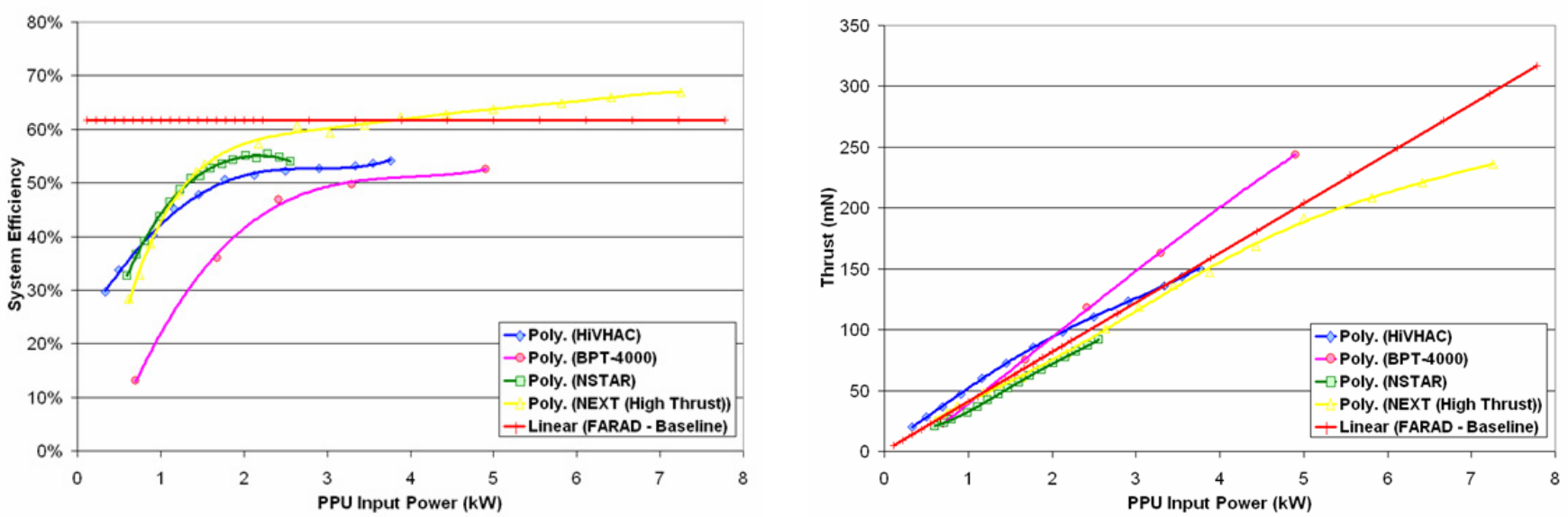

Figure 6. Comparison of efficiency (left) and thrust (right) vs. power for SOA thrusters evaluated.

\section{A. NSTAR}

The NSTAR ion thruster was developed in the early 1990s for use as a primary propulsion system on deep space missions. The NSTAR thruster was successfully used on the New Millennium technology program's Deep Space 1 mission $^{12}$ and it is presently flying on Dawn, which represents the first NASA science mission to use an electric thruster for primary propulsion.

\section{B. NEXT}

The NEXT thruster system is under development as an electric propulsion option for Flagshipclass missions. This thruster offers an evolutionary advance in technology over the SOA NSTAR design. ${ }^{13}$ Relative to NSTAR, the NEXT thruster can operate at a higher maximum power level, which allows for greater thrust, specific impulse, and thrust efficiency. The higher thrust and additional throughput of the NEXT thruster is expected result in a total impulse capability that is greater than the existing NSTAR engine.

\section{HiVHAC}

The High Voltage Hall Accelerator (HiVHAC) thruster development program was originally aimed at producing a $6-8 \mathrm{~kW}$ thruster with moderate $I_{\mathrm{sp}}$ for Flagship-class missions. After the focus shifted from larger missions to smaller Discovery and New Frontiers-class missions, the HiVHAC program adjusted to develop a thruster that would operate at a maximum input power of approximately 3 $\mathrm{kW}$. The effort was specifically aimed at increasing low-power thruster performance while simultaneously reducing the cost for Discovery-class missions using electric propulsion. The HiVHAC thruster can throttle down to operation at a minimum discharge power of approximately 300 Watts. Presently, a $3.6 \mathrm{~kW}$ HiVHAC with the capability to allow in-situ channel replacement is under development with an extended lifetime goal of 30,000 hours operation. ${ }^{14}$

\section{BPT-4000}

The BPT-4000 is a recently flight-qualified Hall thruster developed for use on the Air Force's Advanced Extremely High Frequency Program. The thruster is designed to operate at $3-4.5 \mathrm{~kW}$ and $300-400 \mathrm{~V}$. It is a multi-mode thruster, operating at lower voltage with a higher thrust-to-power ratio for orbit raising maneuvers and then switching to a higher voltage, higher specific impulse mode for station keeping. ${ }^{15}$

Recently, lifetime evaluation testing of the BPT-4000 has been extended to permit the demonstration of a greater propellant throughput capability. In addition, this testing aims to assess the performance of the thruster at lower power operation. The initial flight qualification life testing 
processed approximately $260 \mathrm{~kg}$ of propellant and demonstrated an operational throughput capability of $157 \mathrm{~kg}(150 \%$ margin). Life testing has continued, and the thruster is predicted to have an additional $5,000 \mathrm{hrs}$ of operation remaining.

\section{High Power Thruster Systems}

The issues associated with the use of a pulsed inductive thruster system for high-power propulsion needs can be explored by comparing it with other high-power systems. In this study, comparisons are made with magnetoplasmadynamic, gridded-ion, Hall thruster systems. Each of these is described in turn below. Performance for higher power systems are achieved by using additional thrusters or thruster scaling. The baseline thruster performance parameters used for the high power analyses are shown in Table $\mathrm{V}$.

\section{A. Magnetoplasmadynamic Thruster (MPDT)}

The MPDT is an electric thruster capable of processing significant power levels, $100 \mathrm{~kW}_{\mathrm{e}}$ to $\mathrm{MW}_{\mathrm{e}}$, and producing high thrust densities. While most previous research has been performed using gasfed, multi-megawatt thruster designs ${ }^{16}$, recent work was focused on development of a thruster operating at high efficiency at an input power level of $250 \mathrm{kWe}$. This work was performed under the Advanced Lithium-Fed Applied-field Lorentz Force Accelerator $\left(\mathrm{ALFA}^{2}\right)$ program. ${ }^{17}$ The ALFA ${ }^{2}$ thruster design was motivated by previous data indicating MPDT operation at high efficiency with minimal electrode erosion at relatively low power (order of $100 \mathrm{~kW}_{\mathrm{e}}$ ). The ALFA ${ }^{2}$ target design characteristics and nominal performance values as found in the literature ${ }^{18}$ are presented in Table 5 . These values are based on performance scaling relations for a lithium-fed accelerator, and have yet to be validated with laboratory test data.

\section{B. Herakles Ion Thruster}

The performance for a high power gridded ion engine is based on the development of Herakles thruster. ${ }^{19}$ The Herakles thruster evolved from technology efforts by the Glenn Research Center and the Jet Propulsion Laboratory under the Prometheus Project. While the Herakles thruster fabrication was not completed, the performance is based on data obtained through testing of the HiPEP ${ }^{20}$ and NEXIS ${ }^{21}$ thrusters.

\section{High-Power Hall Thruster}

Several laboratory model Hall thrusters have been tested at very high power levels and the expected performance is very well understood. The near-term projects underway are focusing on higher thrust-to-power Hall systems operating at a discharge power of 20 $\mathrm{kW}$. For this study, the Hall thruster performance is based on the BHT-20K.
Table V. High-power thruster system characteristics used for analyses.

\begin{tabular}{|l|c|c|c|}
\cline { 2 - 4 } \multicolumn{1}{c|}{} & ALFA $^{\mathbf{2}}$ & Herakles & High-Power Hall \\
\hline Propellant & Lithium & Xenon & Xenon \\
\hline Input Power $\left(\mathbf{k W}_{\mathbf{e}}\right)$ & 250 & 28.5 & 20.25 \\
\hline$I_{\mathbf{s p}}$ range $(\mathbf{s})$ & $4500-6200$ & $5500-7000$ & 2750 \\
\hline$T$ range $(\mathbf{N})$ & $\sim 5$ & 0.644 & 1.08 \\
\hline$\eta$ at Full Power $(\mathbf{\%})$ & 60 & 86 & 72 \\
\hline Flow Rate $(\mathbf{m g} / \mathbf{s})$ & $\sim 80$ & $4.2-9.4$ & 40 \\
\hline$T / P\left(N / M W_{\mathbf{e}}\right)$ & 20 & 22.6 & 53.3 \\
\hline Specific Mass $\left(\mathbf{k g} / \mathbf{k W}_{\mathbf{e}}\right)$ & 0.54 & 1.75 & 2.5 \\
\hline
\end{tabular}




\section{Science Mission Applicability}

Three science missions were selected for study in this paper. Two are Discovery class reference missions; the Nereus Asteroid sample return and the Kopff comet rendezvous. A third New Frontiers class mission is the comet surface sample return (CSSR) mission to Temple1. The Nereus mission involves launching with an Earth escape trajectory, using solar electric propulsion to rendezvous with the asteroid, remain at the asteroid for 90 days, and then conduct an Earth flyby for sample release. The Kopff comet rendezvous mission is also designed to launch with an Earth escape trajectory and use SEP to rendezvous with the comet. Last, the CSSR mission also launches to escape, spends 60 days on the comet to collect samples, and then returns back to Earth.

Analysis conducted for these missions were performed using SEPTOP, a calculus of variations low-thrust optimization tool. The missions have been optimized for the greatest delivered mass to the destination. To account for the various thruster systems, the dry mass of the electric propulsion system and a propellant penalty are subtracted from the delivered mass to yield the remaining spacecraft mass as the figure-of-merit. The ion propulsion system (IPS) dry mass includes the thruster, power processing unit, gimbal, and feed system. The propellant penalty accurately accounts for penalties associated with large propellant requirements. If a mission requires additional propellant, it will also require a larger propellant tank and larger propellant residuals and a greater absolute margin. The propellant tank mass is $4.5 \%$ of the propellant requirement while the margins for navigation and trajectory errors and residuals are 5\% and 3.6\% respectively. Last, a mass margin is included with $10 \%$ applied for heritage hardware and 30\% applied for development hardware. Example calculations are shown in Table VI.

Table VI. Example calculations and comparison.

\begin{tabular}{|l|c|c|c|c|c|c|}
\cline { 2 - 7 } \multicolumn{1}{c|}{} & NEXT & HiVHAC & NSTAR & BPT 4000 & FARAD B\&M & FARAD PCBM \\
\hline \# of Operating Thrusters & 1 & 2 & 2 & 2 & 1 & 1 \\
\hline \# of Spare Thrusters for Life & 0 & 0 & 0 & 0 & 0 & 0 \\
\hline \# of Spare Thrusters for Redundancy & 1 & 1 & 1 & 1 & 1 & 1 \\
\hline Total Thrusters & 2 & 3 & 3 & 3 & 2 & 2 \\
\hline Array Power (kW @ 1 AU B.0.L.) & 9 & 9 & 9 & 9 & 9 & 9 \\
\hline Launch Vehicle & Delta I & Delta I & Delta I & Delta II & Delta I & Delta II \\
\hline Trip Time (yrs) & 3.14 & 3.11 & 3.11 & 3.1 & 3.11 & 3.11 \\
\hline C3 (km2/s2) & 1.13 & 1.54 & 2.24 & 2.04 & 0.88 & 0.88 \\
\hline m0 (kg) & 1262.7 & 1251.7 & 1233.5 & 1238.6 & 1269.4 & 1269.4 \\
\hline Mp - Required Throughput (kg) & 159.0 & 192 & 197.2 & 214.2 & 172.9 & 172.9 \\
\hline Throughput Contingency (kg) & 12.9 & 16.5 & 16 & 17.4 & 14 & 14 \\
\hline Total Propellant (kg) & 171.9 & 208.5 & 213.2 & 231.6 & 186.9 & 186.9 \\
\hline Tank Mass (kg) & 7.7 & 9.4 & 9.6 & 10.4 & 8.4 & 8.4 \\
\hline IPS Drymass (kg) & 136.4 & 89.9 & 112.2 & 109.8 & 268.8 & 420 \\
\hline Drymass Contingency (kg) & 40.9 & 27 & 25.6 & 14.5 & 80.6 & 126 \\
\hline Total Wet Mass (kg) & 349.2 & 325.4 & 351 & 355.9 & 536.3 & 732.9 \\
\hline Mass Left at Nereus (kg) & 125 & 125 & 125 & 125 & 125 & 125 \\
\hline Net Delivered Mass (kg) & 788.5 & $\mathbf{8 0 1 . 3}$ & 757.5 & 757.7 & 608.1 & $\mathbf{4 1 1 . 5}$ \\
\hline
\end{tabular}

* Represents S/C, Science Instruments, Entry Capsule, etc.

\section{A. Nereus Asteroid Sample Return}

The Nereus sample return mission has been studied extensively as a Discovery class mission to a near-Earth object. The Nereus orbital properties of a low inclination and moderate eccentricity results in a relatively high optimal specific impulse. For this reason, performance is largely based on the 
propulsion systems efficiencies and specific impulses. An example mission trajectory is shown in Fig. 7.

Even though the mission does not go to a high aphelion, the solar array power produced does change significantly over the course of the mission. The array and thruster power profiles for the FARAD and HiVHAC thrusters are shown in Fig. 8. It is typically observed that low-thrust optimizations typically show a thrust profile with thrust arcs near perihelion, where more power is available for high thrust and high efficiency maneuvers. Though they were omitted for the brevity, the thrust profiles for all of the thruster concepts appeared very similar. Figure 8 illustrates an advantage of the FARAD; namely that it can use all the available power, while the HiVHAC is limited

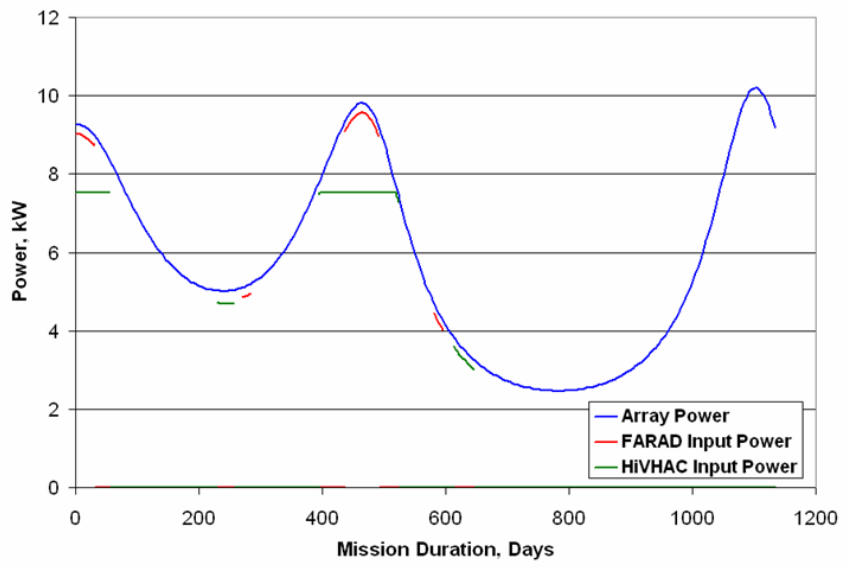

Figure 8. Nereus SR mission power profile.

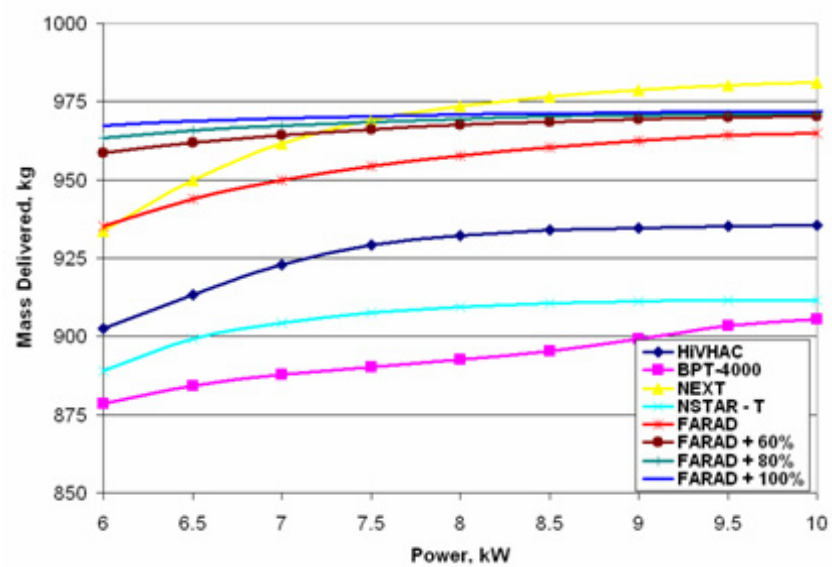

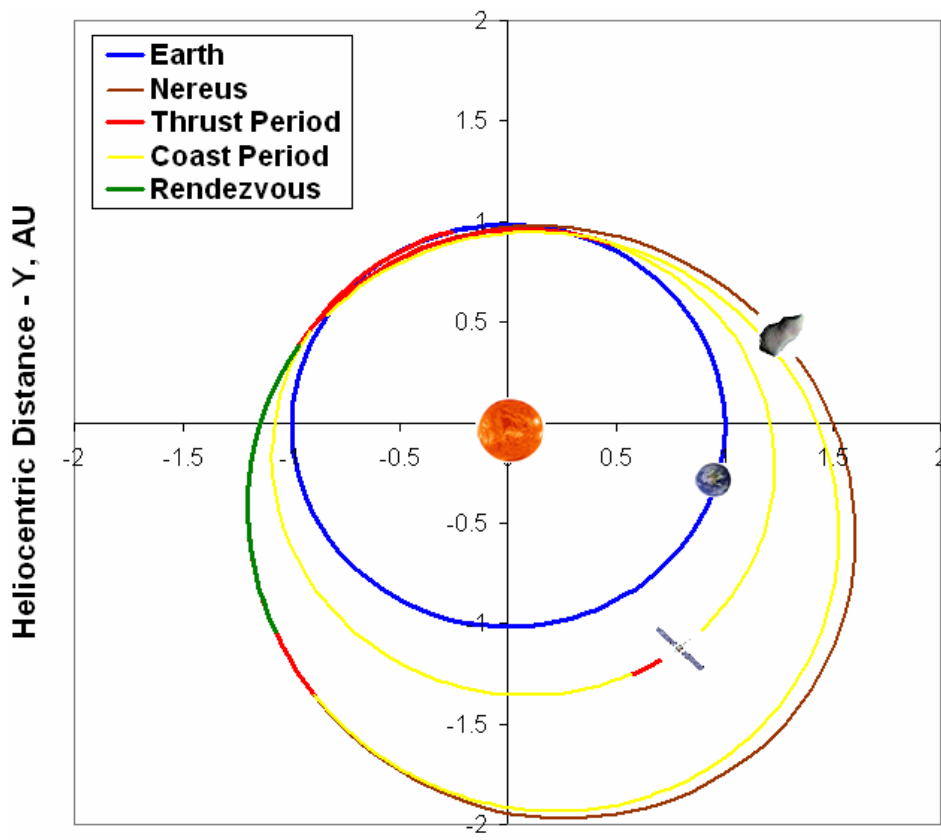

Heliocentric Distance - X, AU

Figure 7. Example Nereus sample return trajectory.

by the thruster's maximum input power. This allows the smallest possible coast period resulting in the minimum maneuver inefficiencies.

Figure 9 illustrates the performance of the various thruster systems. The figure on the left shows the net mass delivered (launch mass less propellant mass) while the figure on the right shows the spacecraft mass remaining after the electric propulsion system (EPS) mass has been removed. It is clear that the FARAD thruster is very competitive with the SOA systems, based on performance alone. The FARAD thruster is a higher performance engine at lower power levels which is critical for low-cost

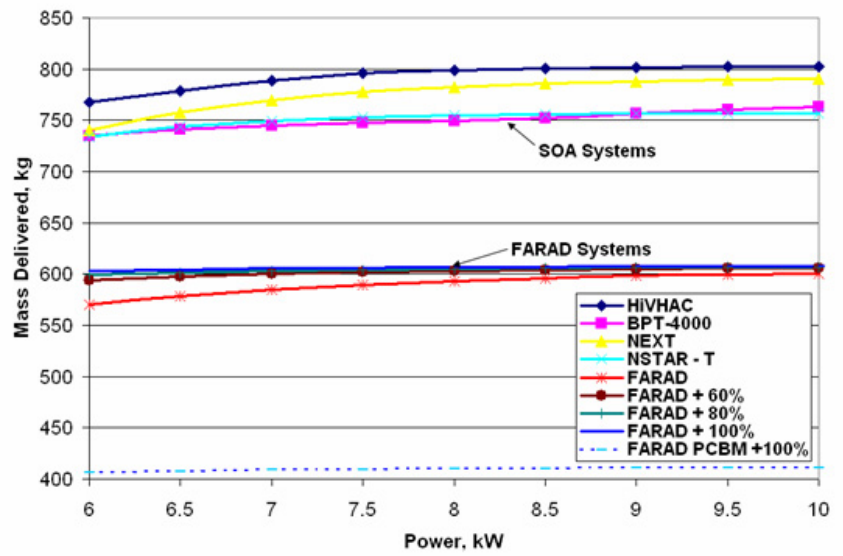

Figure 9. NER Performance versus power without (left) and with (right) EPS Mass removed. 
missions. The FARAD, however, also has the largest dry mass penalty resulting in a net disadvantage when compared to the SOA and development Hall and ion systems. The total number of pulses required of the FARAD to complete the Nereus mission is approximately $1.15 \times 10^{9}$. Because the mass penalty of the PCBM is significantly higher than the B\&M for this mission, the ability for the component life to extend beyond $10^{9}$ pulses without the use of pulsed compression is critical to the applicability of the FARAD to science missions.

\section{B. Kopff Comet Rendezvous}

The Kopff comet rendezvous mission is often used a low-cost reference mission for

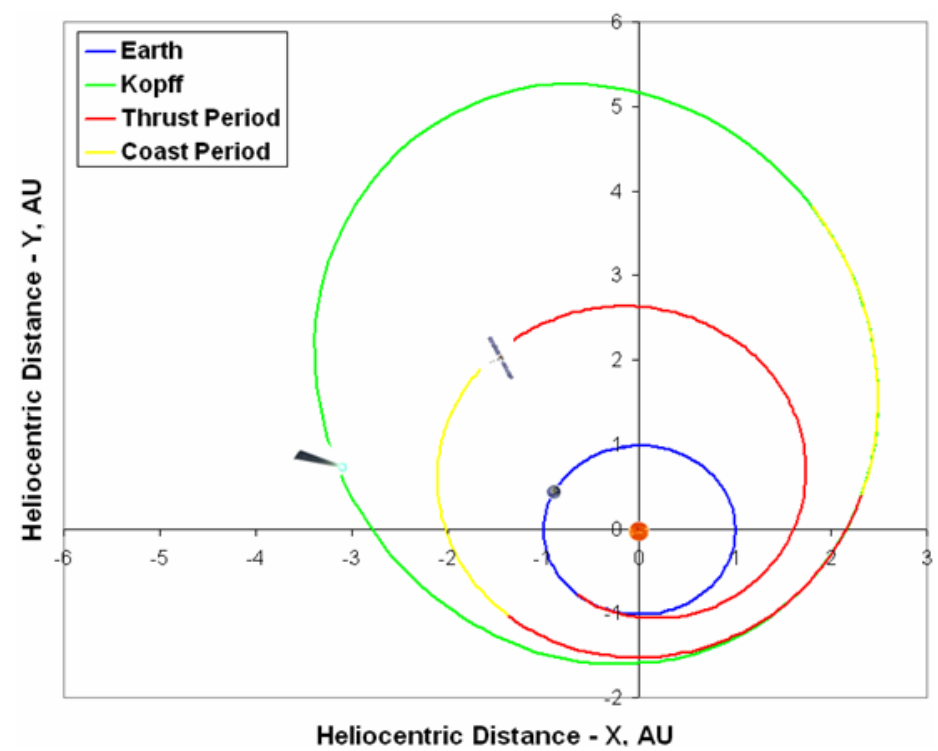

Figure 10. Kopff comet rendezvous trajectory. electric propulsion. The mission is a high AU small body rendezvous which usually favors low-thrust propulsions systems. The Kopff mission is known to have a relatively low (for electric propulsion) optimal specific impulse because of its need for efficient thrust arcs. For the same power level, a higher specific impulse engine will have lower thrust and therefore lower accelerations. An example Kopff rendezvous trajectory is shown in Fig. 10.

With the Kopff rendezvous mission optimizing at a low specific impulse, Hall thrusters and the FARAD are expected to perform well. The power profile shown in Fig. 11 illustrates how the throttleability of the FARAD allows the thruster to operate over the entire range of power available. Only the FARAD and NEXT are able to operate over the entire throttle range with a single thruster.

On performance alone, Fig. 12 (left) shows the FARAD thruster is considerably better than the gridded ion and Hall thrusters. Even without energy recapture, the FARAD outperforms the NEXT

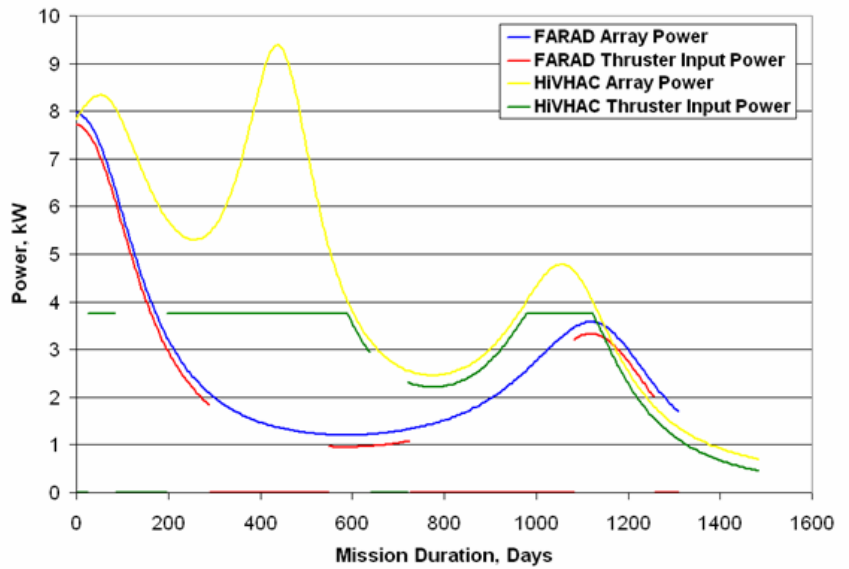

Figure 11. KCR mission power profile. thruster by delivering over $50 \mathrm{~kg}$ in additional net mass. The FARAD also shows an improvement over the SOA electric propulsion systems as the available solar array power decreases. The impediment of the FARAD is again illustrated in Fig. 12 (right). When the EPS mass is included, the SOA systems outperform the FARAD at the higher power levels; that is, SOA systems do better when they have enough power to operate efficiently throughout the mission. The FARAD does still show an advantage to operating at lower overall power levels. It should be noted that while cost is not considered in this study, spacecraft costs are generally proportional to the solar array power. 

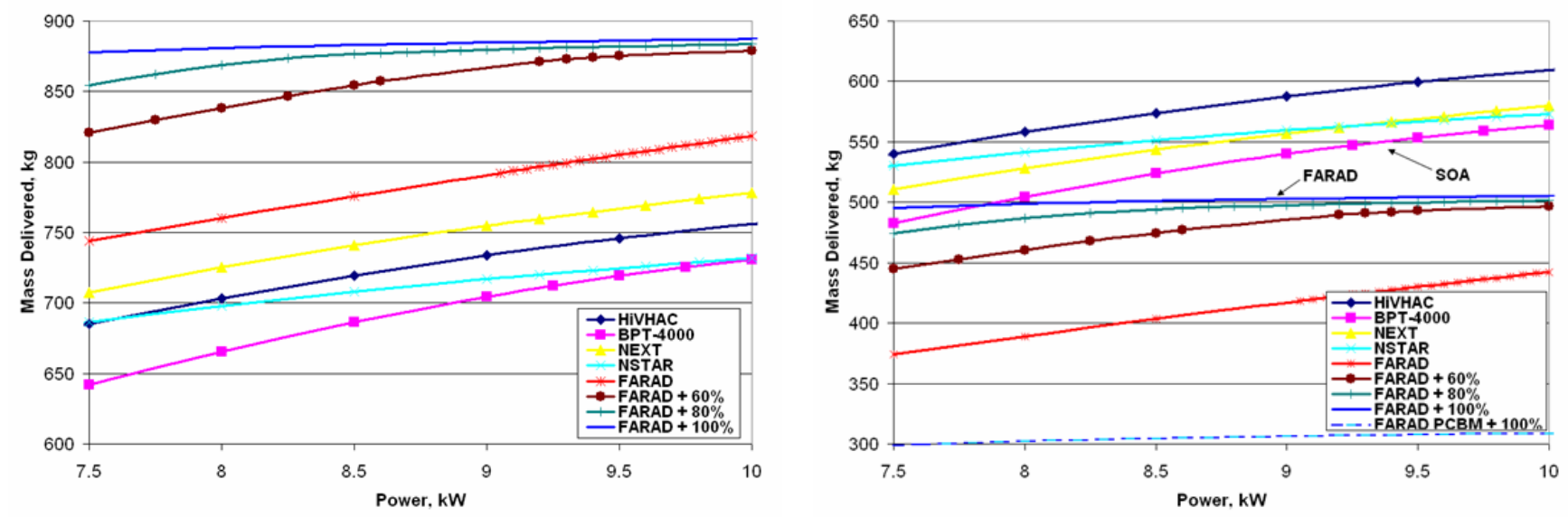

Figure 12. KCR Performance versus power without (left) and with (right) EPS Mass removed.

If the FARAD system dry mass could be reduced to those of the SOA systems, the FARAD would be a significant improvement and possibly cost enabling for Discovery-class missions that optimize to low specific impulses. Because a single FARAD thruster can operate over such a large throttle range, it may be advantageous to try to build single fault tolerance into a single thruster system so that a spare string will not be required. Because the electrodeless thruster does not have an erosion failure mode, single fault tolerance within a single thruster is possible. An ideal case would include using a redundant feed system, capacitors bundled so that single units can be replaced after a failure, and parallel charging PPUs so that only one unit will need to be carried for redundancy. Parallel subsystems will make it difficult to reduce single string system alpha, but can potentially reduce the flight system total IPS mass by eliminating the spare string. If the above system was developed, it could have a feasible dry mass approaching $100 \mathrm{~kg}$ for a $10 \mathrm{~kW}$ system. For the Kopff mission that would be an increase in delivered mass of approximately $75 \mathrm{~kg}$. Based on the Dawn spacecraft, that could translate into an increase in over $300 \%$ more science payload.

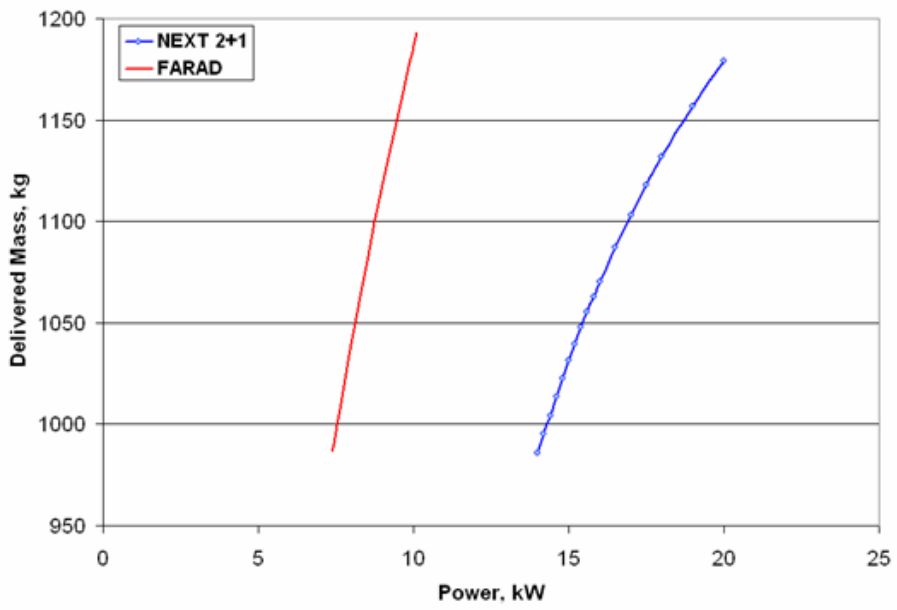

Figure 13. Comparison of FARAD and NEXT for Temple 1 CSSR mission.

\section{Comet Surface Sample Return}

The Temple1 CSSR mission has been studied extensively as a mission ideal for electric propulsion application. ${ }^{22}$ The Templel mission is a challenging low-thrust mission. CSSR missions are known to optimize to low $\mathrm{I}_{\mathrm{SP}}$ and high thrust. ${ }^{23}$ This specific CSSR mission was deemed too difficult for NSTAR because of the thrust required, which necessitated more than four NSTAR engines and a minimum of $15 \mathrm{~kW}$ of power. NEXT is capable of performing the Temple1 CSSR with only two operating thrusters in a $2+1$ configuration and a $15 \mathrm{~kW}$ solar array. Remarkably the FARAD can outperform NEXT as a single thruster with a 50\% reduction in required solar array power. Figure 13 shows the performance of the FARAD and NEXT systems. Both propulsion systems show similar dependency on power, but the FARAD is shifted to the left showing that it can complete the mission with a considerable smaller solar array. The ability to complete the mission at a decreased power arises 
from the thruster's ability to produce appreciable thrust at low input power levels. Figure 14 shows a comparison of two trajectories. The thrust arcs optimize to high AU maneuvers, however the NEXT is not able to operate efficiency below one kilowatt of thruster power.

While the FARAD dry-mass is still higher than that of the NEXT EPS, the solar array reduction will not only make FARAD the highest performing option, but a significantly lower cost option; assuming relatively similar EPS costs. Note that for this more challenging mission, a single FARAD must be able to operate for $4.9 \times 10^{9}$ pulses.
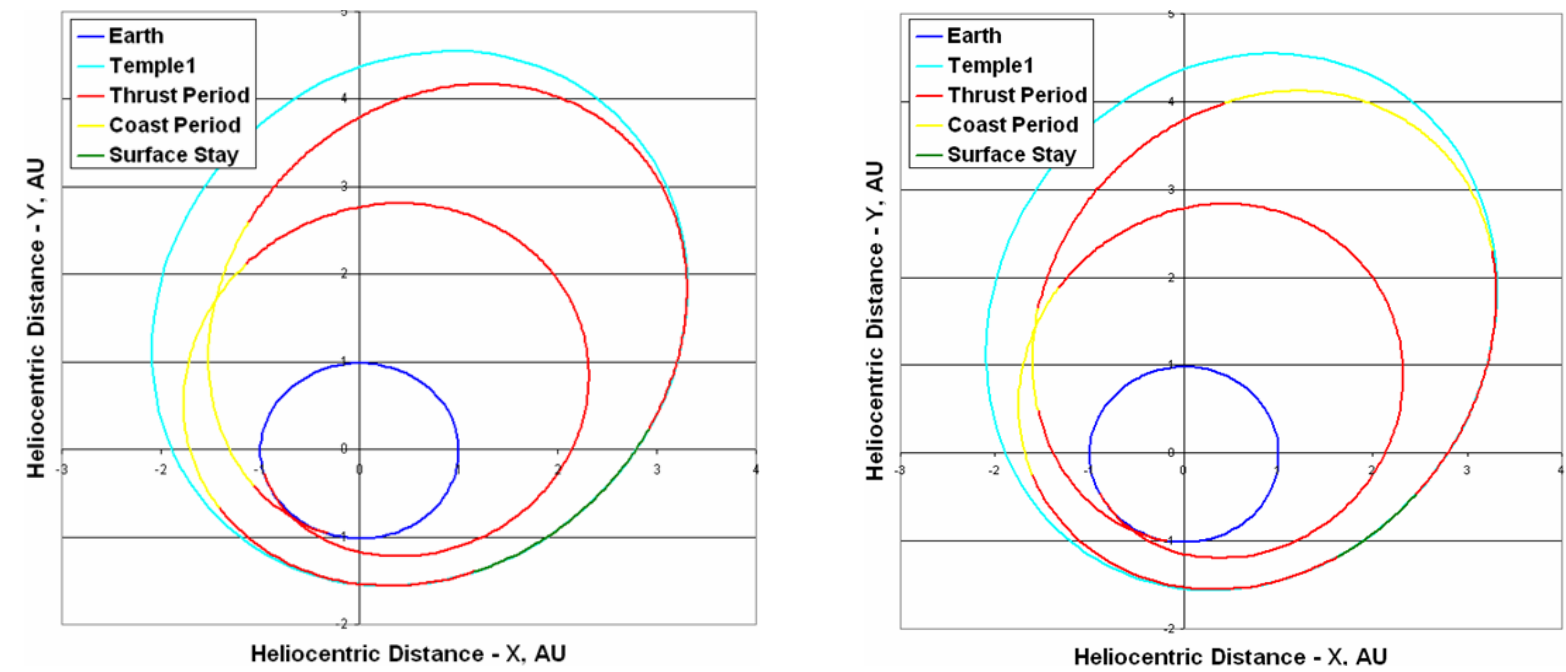

Figure 14. Comparison of FARAD (left) and NEXT (right) trajectory for Temple 1 CSSR Mission.

\section{Exploration Mission Applicability}

The two exploration (high-power) missions selected for study are a cargo mission to Mars and a cargo mission to the moon with oxygen return. The Mars cargo mission is based on the need to deliver substantial mass to Mars prior to the manned mission planned for the 2030 timeframe. The lunar cargo tug mission is based on the exploration objective of building a lunar base, coupled with the concept of using lunar oxygen as fuel for the human Mars mission. It is believed that using oxygen produced from the moon will significantly reduce the cost of the crewed mission to Mars by reducing the initial mass to low Earth orbit (IMLEO).

Analyses for these exploration missions were performed using Copernicus and SECKSPOT. Typically, exploration missions such as cargo to the moon or Mars are only dependent on the efficiency and specific impulse at full power. The mission $\Delta \mathrm{V}$ does not vary much based on the thruster performance or throttle-ability because the available power does not vary significantly during the missions. 


\section{A. Mars Cargo Mission}

For the Mars cargo mission, the power source can be either solar or nuclear. The only performance variation due to the power source is based on the power system alpha (in $\mathrm{kg} / \mathrm{kW}$ ). The figure-of-merit for these analyses is the required IMLEO to deliver the necessary cargo. The Mars cargo mission will be traded for a small and a large cargo mission. The baseline mission is to deliver 20 metric tons with a $250 \mathrm{~kW}$ power source. The gridded-ion and Hall thrusters again have the advantage of decades of investment. Recently, the NASA Prometheus Program invested heavily into gridded-ion engines and on a lesser scale, high power Hall, MPD, and pulsed inductive thrusters. Figure 15 shows

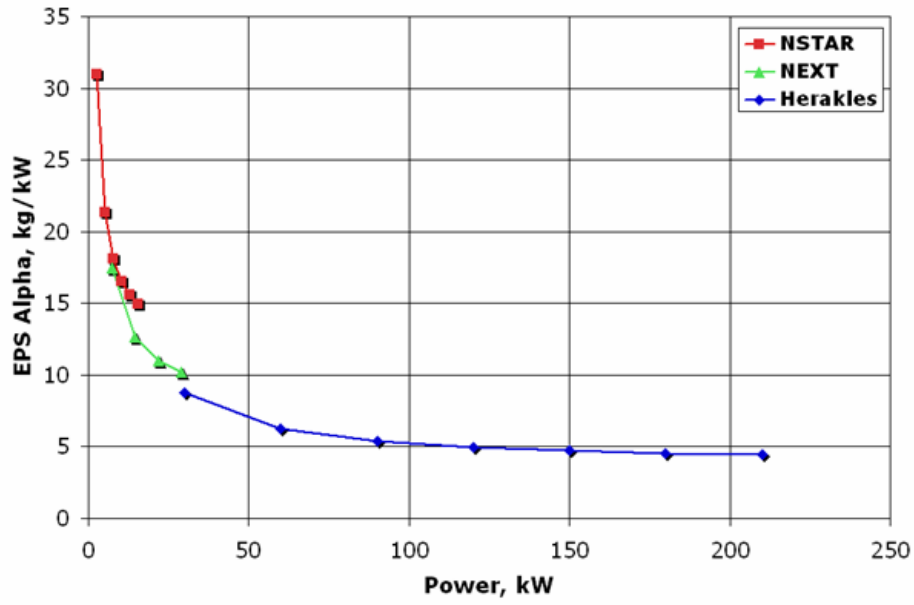

Figure 15. Predicted system alpha for gridded-ion engines. the progress made on the system alpha for gridded-ion engines. JPL performed work the NEXIS thruster while GRC focused on HIPEP. The two engines were later combined to develop a high efficient, long life, low system alpha thruster, Herakles. The cargo trajectory from Earth to Mars is typically tangential to the sun line with a short coast period. An example trajectory is shown in Fig. 16.

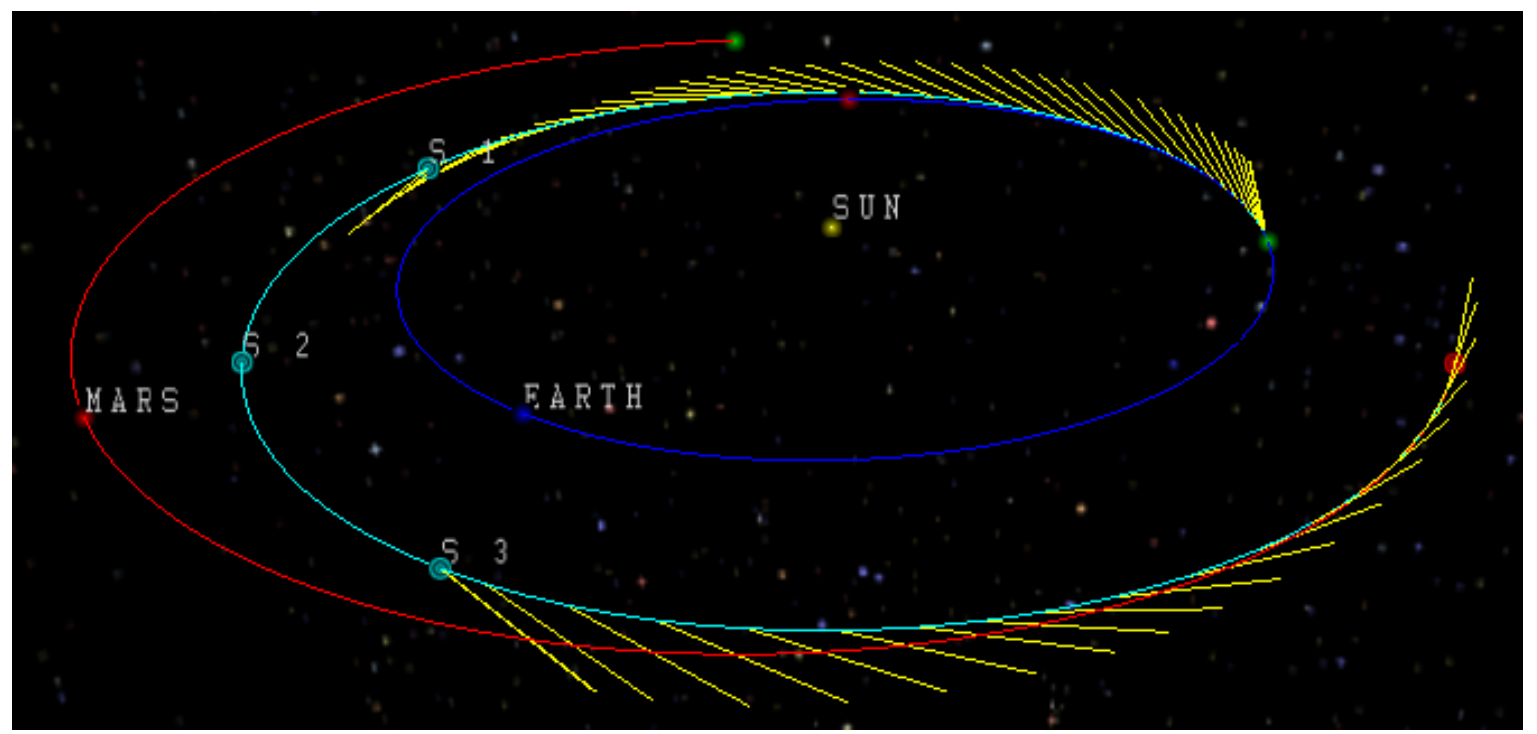

Figure 16. Representative low-thrust Earth to Mars cargo mission.

Whether a solar or nuclear power system is used, the mass is considered equal and independent from the EPS. The results are shown in Fig. 17 for a delivery of 20,000 kg, and illustrate that the higher specific impulse systems outperform the baseline FARAD. Even with a higher Isp FARAD, the dry mass of the IPS is more than both SOA systems and the MPD. The efficiency gain from using energy recapture does reduce the propellant requirement, but steady-state, high-power systems counter this by having low propellant requirements because they are efficient. Packaging was not considered in this study, however previous studies have shown that volume constrained launch vehicles would favor a pulsed device or MPD due to the exit area required to produce the thrust from gridded-ion and Hall thrusters. $^{24}$ 


\section{B. Lunar Cargo Mission}

The decision to establish a base on the surface of the moon has engendered high interest in lunar cargo missions. While the baseline plan is to use chemical systems to deliver cargo, electric propulsion systems have been continuously recommended as the only sustainable method to deliver significant payloads to the moon. The baseline trajectory for a lunar cargo mission is to begin spiraling out from LEO until exiting the Earth's sphere of influence and then spiral down towards the moon. An example trajectory is shown in Fig. 18. All of the spirals are not shown in the figure, rather a representative path.

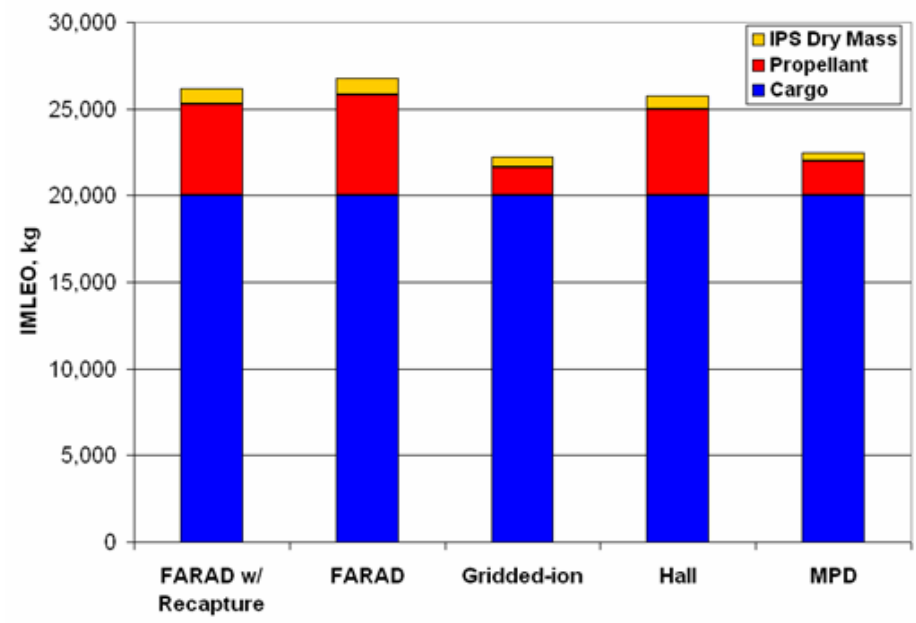

Figure 17. IMLEO comparison for Mars cargo.

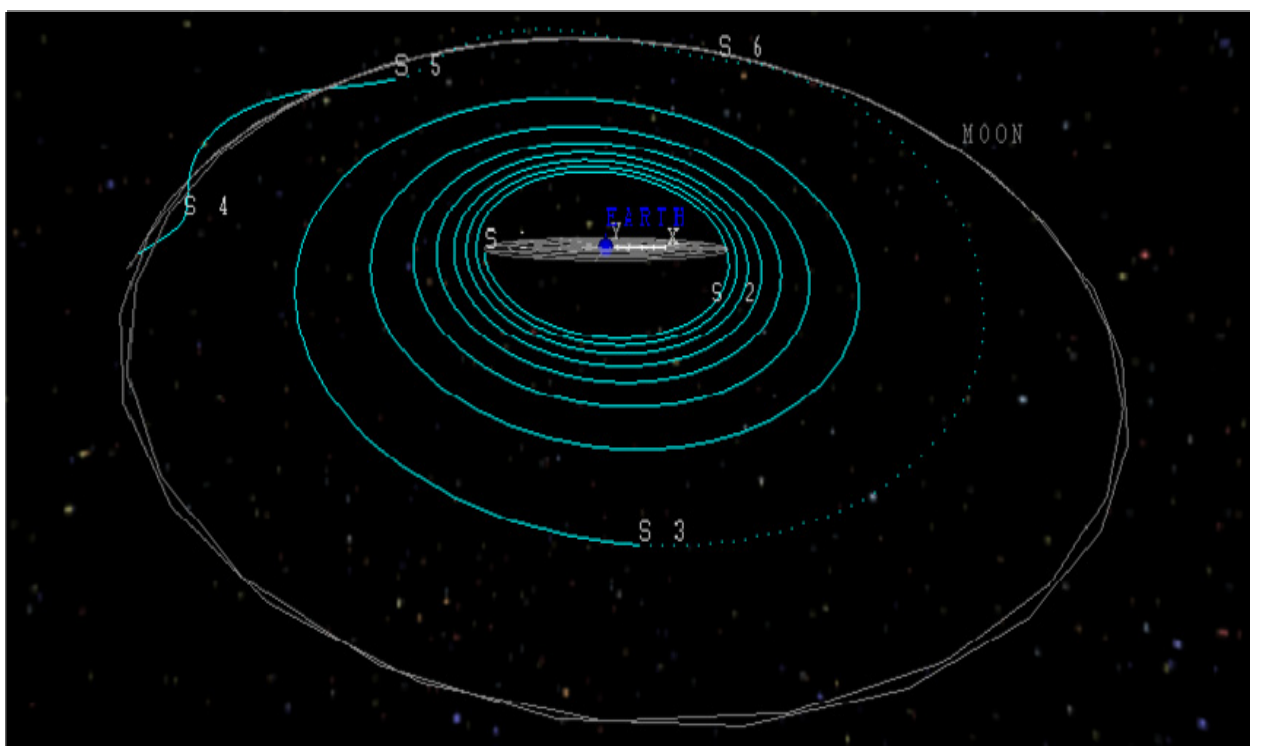

Figure 18. Representative low-thrust Earth to Moon trajectory.

One of the drawbacks to using electric propulsion for exploration cargo missions is the long trip times associated with low-thrust propulsion. Without specific cargo requirements, the baseline mission was to deliver approximately $50 \mathrm{MT}$ to the moon, and to be able to perform the mission on an annual basis. Based on the trip-time, a sufficiently high power electric propulsion tug can meet those objectives. For comparison purposes, a $1 \mathrm{MW}$ system was used. The mission $\Delta V$ 's and trip times are partially dependent on the thruster system performance. The lower specific impulse (higher thrust) systems typically require a lower $\Delta \mathrm{V}$ to complete the mission. This variation in $\Delta \mathrm{V}$ is common and due to the reduction in gravity losses associated with higher accelerations during the LEO spiral out phase. The gridded-ion engine does have a

Table VII. One way trip time values for lunar transfer.

\begin{tabular}{|l|c|}
\cline { 2 - 2 } \multicolumn{1}{c|}{} & Trip Time (days) \\
\hline FARAD w/ Recap & 70 \\
\hline FARAD & 95 \\
\hline Gridded-ion & 167 \\
\hline Hall & 66 \\
\hline MPD & 213 \\
\hline
\end{tabular}


higher specific impulse, but also operates at a higher efficiency that the FARAD. As shown in Table VII, the trip times are as expected; the Hall and FARAD systems perform a relatively fast transfer, while the lower thrust gridded-ion and MPD engines required significantly longer times to deliver their cargo. The one megawatt power source is sufficient to deliver the cargo within a single year; however, the gridded-ion system and especially the MPD are challenged to complete the return trip within the same year. Return transfers are shorter due to the lack of cargo.

Several studies have indicated a significant cost savings through the use of propellant produced on the moon. One possible cost reduction of a human Mars mission is to deliver the liquid oxygen propellant from the moon to LEO for the Earth Departure Stage (EDS). Therefore, it may be advantageous to use the electric propulsion tug to ferry cargo to the moon and also transport propellant back to LEO. In order for a cargo system to be able to transfer cargo back from the moon, the initial vehicle at Earth must carry enough propellant for the complete roundtrip. One advantage of using the FARAD is the ability to use in-situ propellants. The performance gain is two fold; the propellant and associated dry mass for the return trip do not have to be included at Earth departure, and the propellant produced on the moon will be "bought" at a reduced cost. The results in Fig.19 show the initial mass to low earth orbit required to deliver the necessary cargo.
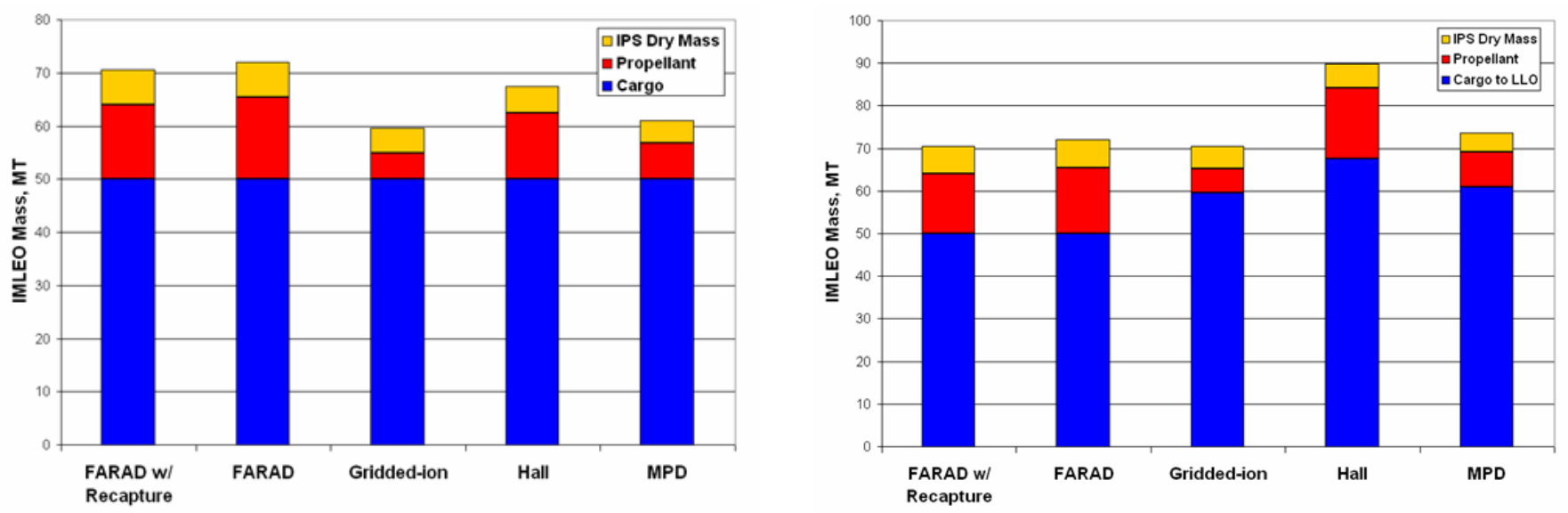

Figure 19. IMLEO requirements for one-way (left) and round-trip (right) lunar cargo mission.

For a one-way mission, the reduced IPS system masses and propellant masses of the higher specific impulse options show significantly better performance. If a round-trip is considered, the in-situ capabilities of the FARAD make it competitive with the SOA systems. The niche for the FARAD for lunar cargo would be the desire to complete roundtrip missions on the order of 6 months. The FARAD can offer trip times comparable to the higher thrust-to-power options and still have a reasonable IMLEO. Also, the current NASA program is operating on a "Go-AsYou-Pay" system where missions are completed only as money becomes available. This is a different approach from choosing a mission and paying whatever it costs. By using a "Go-As-You-Pay" comparison of the various systems it becomes clear that the FARAD does have some advantages over SOA systems. Figure 20 shows how much

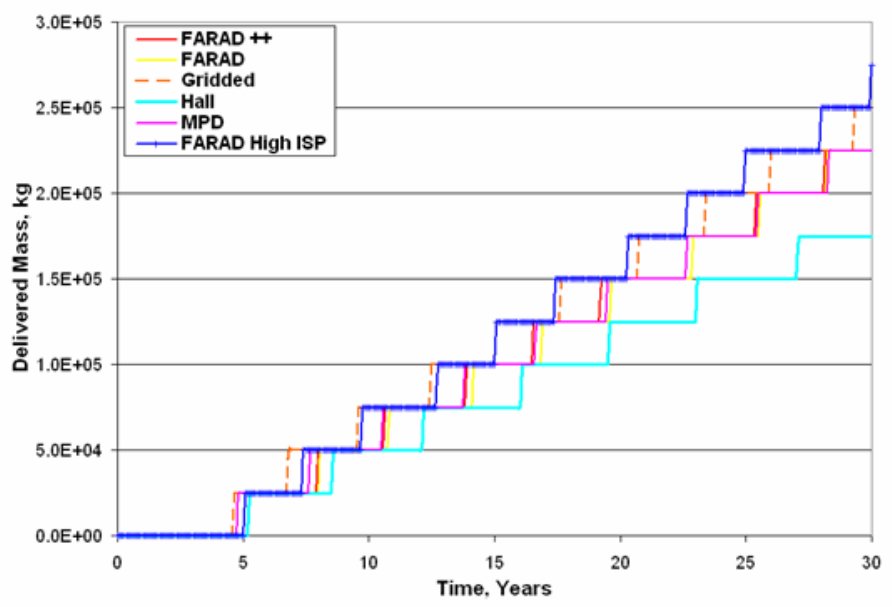

Figure 20. Cargo delivered using "Go-As-You-Pay." 
cargo can be delivered to the moon simply assuming a launch budget of $\$ 120 \mathrm{M}$ per year and a launch cost of $\$ 10,000$ per $\mathrm{kg}$. With this cost constraint, it takes approximately five years before any system is completed in-orbit and ready to begin delivering cargo. The gridded-ion system is able to make its first delivery in just less than five years, with the FARAD and Hall systems completing their first cargo delivery approximately nine months later. Because of the reduced re-supply costs of the FARAD, over time it emerges as the highest performing system by cost. The FARAD thruster can be operated at a higher specific impulse to give the added mass ratio benefit, and shows a markedly better performance than any of the other systems. The in-situ capability of the FARAD can make it the most sustainable thruster system.

\section{Near-Earth Applications}

Unlike the electric propulsion applications previously discussed, there is considerable use of electric propulsion in near-Earth space. Typically the thrusters are used for North-South station-keeping to prolong the life of GEO satellites. EP systems have also been used for orbit topping and full GTO-toGEO EP-powered transfers are imminent with the launch of the Air Force EHF asset as well as foreign launch market demands. The FARAD GTO-to-GEO capability is compared to the existing and nearterm Hall and gridded ion engine performances provided in Fig. 21.
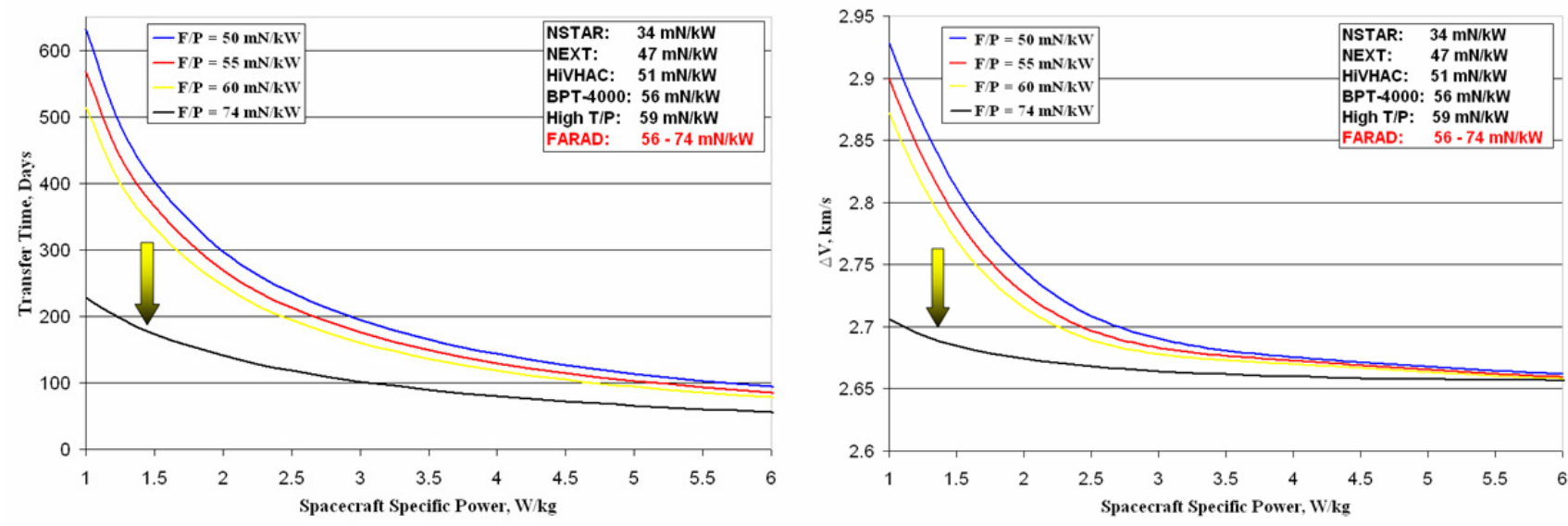

Figure 21. FARAD comparison to Hall and gridded ion thruster for GTO-to-GEO transfers.

The transfer time and $\Delta \mathrm{V}$ for each low-thrust spiral phase has been calculated using SEPSPOT. SEPSPOT is a Glenn Research Center variation of Solar Electric Control Knob Setting Program by Optimal Trajectories (SECKSPOT). ${ }^{25}$ The program is a low-thrust optimizer that calculates the time optimal geocentric trajectory with attitude constraints. The attitude constraint causes power to become a function of both thrust direct and sun directions, and the time optimal thrust direction is a complex function of the primer vector direction. The code has capabilities to determine array degradation through an internal radiation model and account for startup delays after emerging from shadow, however; the degradation and delay factors are not current with state-of-the-art technology. Because of the advancement in array technologies and xenon thruster ignition delays, those features of the code have been turned off. A user may choose to add sufficient Beginning of Life (BOL) array margin to account for array degradation, but GTO-to-GEO degradation should be minimal with a worst case estimate of only $10 \%$. The code always has the ability to determine occultation effects, but since the occultation delay is dependent on acceleration, launch date and inclination, and there is difficulty in achieving convergence with this feature enabled in the code; is has been switched off for this study. 
The analyses also do not include occultation delays to allow for differentiation of propulsion system performance effects. Typically the delays can be easily minimized to values near $2 \%$ of the total transfer time, while zero occultation is possible for transfer times less than 160 days. The worst case occultation effect would occur when the sun line is aligned with the line of nodes and shadowing occurs at apogee when the spacecraft is traveling the slowest. These worst case effects could represent approximately $7 \%$ of the total transfer time.

For those preferring shorter transfer time over performance, 1000s specific impulse is possible; efficiencies currently only allow approximately $70 \mathrm{mN} / \mathrm{kW}$ with corresponding transfer times just under 100 days. There is a performance gain for steady-state thruster when increasing from 1000s to 2000s without a significant increase in transfer time because of the increase in efficiency over this range. However, the performance increase from 2000s to 4000s specific impulse comes at a significant transfer time penalty because there is very little increase in efficiency over this range. Also, the $\Delta \mathrm{V}$ increase associated with changing from a higher thrust to higher specific impulse thruster is approximately 250 $\mathrm{m} / \mathrm{s}$, which partially diminishes the benefit of operate at higher specific impulse. Results from the FARAD are shown in Fig. 21. The comparison clearly indicated there is significant gain to be had from the FARAD thruster due to its ability to operate at high efficient at higher thrust. The NSTAR and NEXT gridded ion engines operate at a thrust-to-power of 34 and $47 \mathrm{mN} / \mathrm{kw}$ respectively. Hall thrusters can operated efficiently at a higher thrust-to-power of $56 \mathrm{mN} / \mathrm{kW}$, but only have expectations of approaching $60 \mathrm{mN} / \mathrm{kW}$ above 2000s specific impulse. Efficiencies of various power level and fidelity Hall and gridded-ion engines are shown in Fig. 22. The FARAD has the potential to operate from 56$74 \mathrm{mN} / \mathrm{kW}$ depending on the energy recapture efficiency. This potential is beyond that of SOA technologies and may offer realistic opportunity to approach GTO-to-GEO transfer on the order of 30 days.

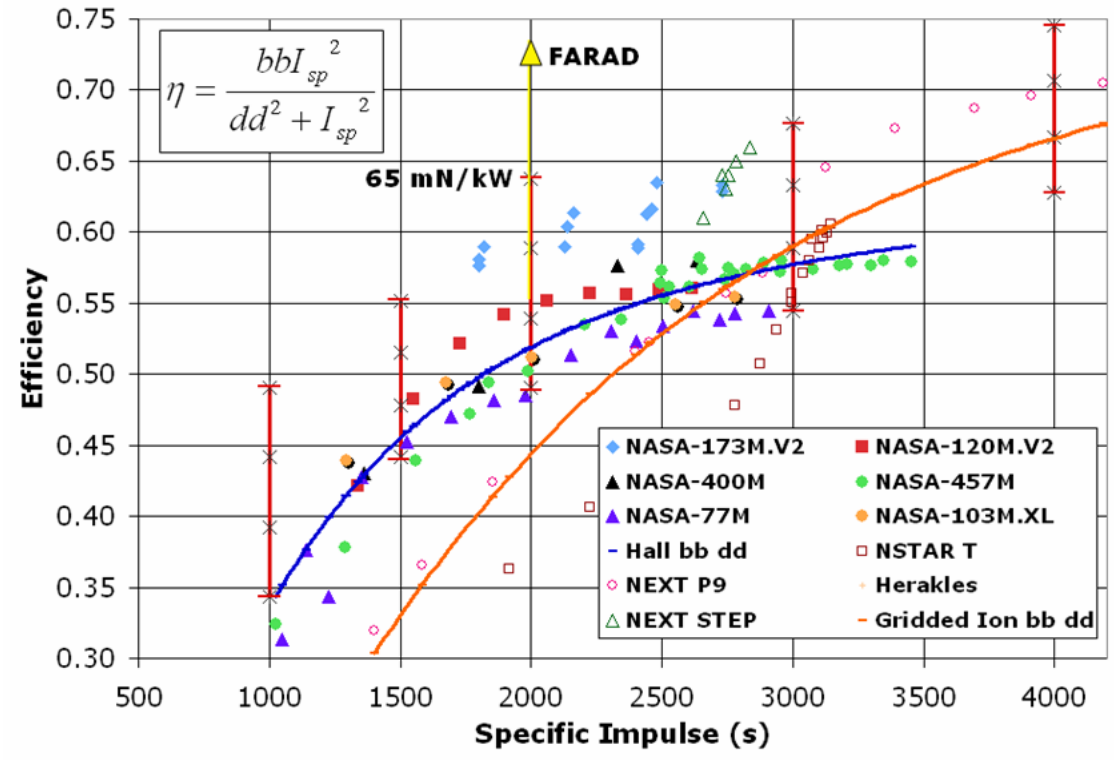

Figure 22. Efficiency versus power for Hall and gridded ion thrusters.

Another observation critical when choosing a propulsion system is that increase in specific impulse does not often yield the full gain one might anticipate using Tsiolkovsky's rocket equation. The transfer $\Delta \mathrm{V}$ is also a function of the spacecraft thrust, or more accurately, acceleration. Using a higher thrust propulsion system can reduce the $\Delta \mathrm{V}$ requirement by either minimizing losses or by operating with more 
efficient maneuvers. Examples include gravity losses, raising perigee at apogee, or plane changes at apogee. This effect is also illustrated in Fig. 21 (right).

While the potential for FARAD is significant, transfer time is purely a function of the spacecraft acceleration. The acceleration is a function of the thrust-to-mass, or generalized by the power-to-mass of the vehicle. Because the mass of the baseline FARAD system is considerable higher than that of the SOA systems, the use of FARAD will shift the performance on the specific power curve; lowering the overall performance expectation. For larger COMSATs, the shift may be acceptable. Because the mass and power of commercial satellites are both increasing, an $18 \mathrm{~kW}$ spacecraft with a $3000 \mathrm{~kg}$ GEO mass may only have a specific power shift on the order of $0.2 \mathrm{~W} / \mathrm{kg}$ using the FARAD and still considerable outperform the SOA propulsion systems. Clearly the benefit of higher power and high mass payloads will minimize the penalty of the heavier FARAD system, but as mission leverage the electric propulsion system, such as LEO-to-GEO, the performance gains will be amplified.

\section{Conclusions}

The analyses for this study are generic enough to be applicable for general pulsed propulsion systems. Overall, the FARAD system does show significant advantage over SOA for several mission applications. The primary conclusions are as follows:

1. The performance of the FARAD at low power is considerably higher that SOA systems.

2. Energy recapture did not lead to significant gains for science mission unless the system was power starved.

3. At high-power, the efficiency and life-times of steady-state systems is significantly higher than existing pulsed devices.

4. The FARAD system dry mass is a hindrance to its applicability at low power.

5. System dry mass reductions are generally more valuable than improvements to thruster performance.

6. Due to the low energy per pulse nature of the FARAD (opposed to the PIT), the benefit of a PCBM is likely outweighed by the additional increase in system dry mass.

7. The powertrain for a pulsed system must be capable of $>10^{9}$ pulses.

8. A single thruster string with built-in fault tolerance is recommended to reduce total FARAD IPS dry mass.

9. A significant advantage for FARAD, especially in the lunar cargo mission, is its capability to utilize in-situ propellants.

10. The FARAD has the potential for higher efficiency, high thrust-to-power transfers than SOA technology projections.

\section{References}

1 Dailey, C. L., and Lovberg, R., H., The PIT MkV Pulsed Inductive Thruster, Technical Report NASA CR-191155, TRW Systems Group, July 1993.

2 Choueiri, E. Y., and Polzin, K. A., "Faraday Acceleration with Radio-frequency Assisted Discharge (FARAD)," Journal of Propulsion and Power, Vol. 22, No. 3, May-June 2006.

3 Eskridge, R.H., Fimognari, P.J., Martin, A.K., and Lee, M.H., "Design and Construction of the PT-1 Prototype Plasmoid Thruster," AIP Conf. Proc., edited by M.S. El-Genk, Vol. 813, pp. 474, 2006.

4 Polzin, K.A., "Comprehensive Review of Planar Pulsed Inductive Plasma Thruster Research and Technology," J. Propuls. Power, submitted for publication, July 2008.

5 Polzin, K.A., Rose, M.F., and Miller, R. "Laboratory-Model Integrated-System FARAD Thruster," in $44^{\text {th }}$ AIAA/ASME/SAE/ASEE Joint Propuls. Conf., Hartford, CT, July 2008. AIAA Paper 2008-4821.

6 Bernardes, J. and Meryman, S., "Parameter analysis of a single stage induction mass driver," In M.F. Rose and P.J. 
Turchi, editors, Proceedings of the 5th IEEE International Pulsed Power Conference, pp. 552-555, Arlington, VA, 1985. Paper PI-27.

7 Owens, T. L., “A Pulse-Compression Ring Circuit for High-Efficiency Electric Propulsion,” Review of Scientific Instruments, Vol. 79:034701-1 - 034701-10, 2008.

8 Polzin, K.A., "Scaling and Systems Considerations in Pulsed Inductive Plasma Thrusters", IEEE Transactions on Plasma Science, in press, Aug. 2008.

9 Lovberg, R. H. and Dailey, C. L., “A PIT Primer,” Technical Report 005, RLD Associates, Encino, CA 1994.

10 Personal communication with Radiance Technologies, Robert Miller, March, 2007.

${ }^{11}$ Baggett, R., et. al., "In-Space Propulsion Solar Electric Propulsion Program Overview of 2006," 42 AIAA/ASMA/SAE/ASEE JPC, Sacramento, CA, July 2006.

12 Polk, J. E., et. al., "Performance of the NSTAR Ion Propulsion System on the Deep Space One Mission," $39^{\text {th }}$ Aerospace Sciences Meeting and Exhibit, Reno, NV, January 2001.

${ }^{13}$ Patterson, M., et. al., "NEXT: NASA's Evolutionary Xenon Thruster," $38^{\text {th }}$ AIAA/ASMA/SAE/ASEE JPC, Indianapolis, IN, July 2002.

${ }^{14}$ Manzella, D., and Oh, D., "Hall Thruster Technology for NASA Science Missions," $41^{\text {st }}$ AIAA/ASMA/SAE/ASEE JPC, Tucson, AZ, July 2005.

${ }^{15}$ De Grys, K., et. al., "BPT-4000 Multi-Mode 4.5 kW Hall Thruster Qualification Status," AIAA-2003-4552, Huntsville, AL, July 2003

${ }^{16}$ Choueiri, E.Y. and Ziemer, J.K., "Quasi-Steady Magnetoplasmadynamic Thruster Performance Database," Journal of Propulsion and Power, Vol. 17, No. 5, pp. 967, 2001.

${ }^{17}$ Cassady, R.J., et al., "Recent Advances in Nuclear Powered Electric Propulsion for Space Exploration," Energy Conversion and Management, Vol. 49, pp. 412, 2008.

${ }^{18}$ St. Rock, B., Blandino, J.J., Anderson, K.R., and Polk, J.E., "Thermal Analysis of a Lithium Vaporizer for a High-Power Magnetoplasmadynamic Thruster," Journal of Propulsion and Power, Vol. 22, No. 5, pp. 1098, 2006

19 Patterson, M. J., Elliott, F., Malone, S. P., Soulas, G. C., Goebel, D., and Sengupta, A., "Herakles Thruster Development for the Prometheus JIMO Mission," 41 ${ }^{\text {st }}$ AIAA/ASMA/SAE/ASEE JPC, Tucson, AZ, July 2005.

${ }^{20}$ Williams, G. J., Foster, J. E., Hickman, T. A., Malone, S. P., and VanNoord, J. L., "Performance Characterization of a 21 kW High Specific Impulse Ion Thruster," 41 $1^{\text {st }}$ AIAA/ASMA/SAE/ASEE JPC, Tucson, AZ, July 2005.

${ }^{21}$ Polk, J. E., Goebel, D. M., Snyder, J. S., Schneider, A. C., Johnson, L. K., and Sengupta, A., "Performance and Wear Test Results for a $20 \mathrm{~kW}-$ Class Ion Engine with Carbon-Carbon Grids," $41^{\text {st }}$ AIAA/ASMA/SAE/ASEE JPC, Tucson, AZ, July 2005.

${ }^{22}$ Cupples, M., Woo, B., and Coverstone, V., "Application of Solar Electric Propulsion to a Comet Surface Sample Return Mission," 40 ${ }^{\text {th }}$ AIAA/ASMA/SAE/ASEE JPC, Ft. Lauderdale, FL, July 2004.

${ }^{23}$ Dankanich, J. W., and Polsgrove, T., "Mission Benefits of Gridded Ion and Hall Thruster Hybrid Propulsion Systems, $42^{\text {nd }}$ AIAA/ASMA/SAE/ASEE JPC, Sacramento, CA, July 2006.

${ }^{24}$ Frisbee, R., "Evaluation of High-Power Solar Electric Propulsion Using Advanced Ion, Hall, MPD, and PIT Thrusters for Lunar and Mars Cargo Missions," 42 ${ }^{\text {nd }}$ AIAA/ASMA/SAE/ASEE JPC, Sacramento, CA, July 2006.

${ }^{25}$ Sackett, Lester L, Malchow, Harvey L., and Edelbaum, Theodore N., "Solar Electric Geocentric Transfer with Attitude Constraints: Analysis", NASA CR-134927, August, 1975. 